\section{"Da sind einige echte Kracher dabei“}

Praktisch, interaktiv und curricular: Der 12. RadiologieKongressRuhr (RKR) punktet mit einem breiten Programm, welches das vielfältige Spektrum der Radiologie präsentiert. Kongresspräsident Prof. Dr. Stefan Rohde berichtet im Interview, welche Neuigkeiten der größte radiologische Regionalkongress Deutschlands vom 7. bis 8 . November 2019 zu bieten hat und warum sich der Besuch lohnt.

\section{Professor Rohde, was zeichnet den 12. RadiologieKongressRuhr aus?}

Der RKR bietet ein breites Fortbildungsprogramm - für Kollegen und Kolleginnen in der radiologischen Weiterbildung ebenso wie für erfahrene Radiologinnen und Radiologen. Sein breites Programm mit Vorträgen hervorragender Referenten aus dem ganzen Bundesgebiet zeigt: Der RKR ist mittlerweile auch überregional fest im radiologischen Kalender etabliert. Neu ist in diesem Jahr der curriculare Aufbau: Die verschiedenen radiologischen Bereiche sollen - geordnet nach anatomischen Regionen - kontinuierlich bearbeitet werden. Die Kongresse der nächsten Jahre werden passgenau auf den Inhalten der Vorjahresprogramme aufbauen.

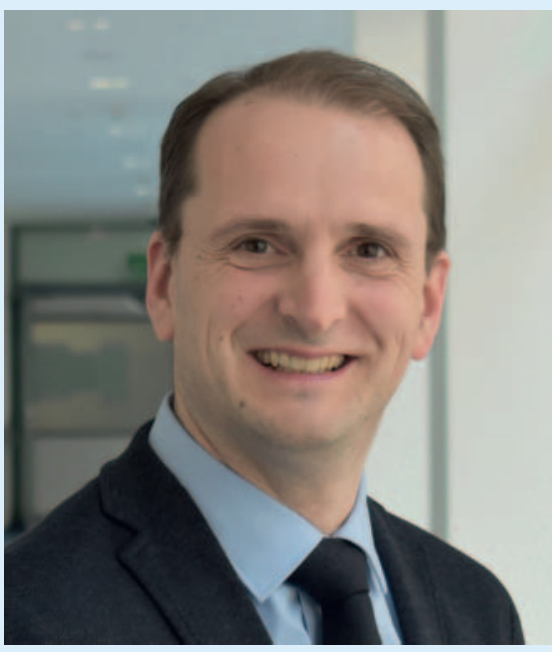

Prof. Dr. Stefan Rohde, Klinikum Dortmund

Großen Organregionen, wie z. B. der abdominellen oder der thorakalen Bildgebung, wird entsprechender Raum durch Schwerpunktsitzungen gegeben, um die verschiedenen Pathologien auch in der nötigen Tiefe vorstellen zu können. Aber auch kleinere Fächer und Themen werden berücksichtigt, wie z. B. die Kinderradiologie, die Urodiagnostik oder die Kopf-Hals-Bildgebung.
Dürfen sich die Besucherinnen und Besucher auch auf Veranstaltungs-Premieren freuen?

Allerdings! Zu ersten Mal präsentieren wir die Veranstaltung „Meine schönsten Fehler“. Hier werden Experten über ihre radiologischen Fehldiagnosen, irreführenden Bildinterpretationen und andere „pitfalls“ berichten und mit den Teilnehmern diskutieren. Aus Fehlern lernt man bekanntlich am besten. Außerdem soll es auf dem RKR wieder praktisch werden, weshalb wir die Simulatoren-Kurse wieder mit ins Programm aufgenommen haben. Diese Kurse eignen sich insbesondere für Berufsanfänger, um erste interventionelle Erfahrungen zu sammeln. Aber auch für fortgeschrittene Interventionalisten ist dieser Kurs interessant, da anspruchsvolle Behandlungsszenarien mit unterschiedlichen Materialen in einem sehr realistischen „Angiosetting“ simuliert werden können. Und es wird ein interaktiver Kongress, dank TED-Abstimmungen. Die Besuchenden können sich erstmals mit dem eigenen Smartphone an vielen Sessions direkt beteiligen. 
Welche aktuellen Themen hält der RKR bereit?

Auf dem diesjährigen RKR planen wir eine spezielle Sitzung für aktuelle berufspolitische Themen und wir werden die Neuerungen durch das Strahlenschutzgesetz genauer betrachten, speziell mit Fokus auf niedergelassene Radiologinnen und Radiologen, die keinen MPE beschäftigen können. Und wir schauen auf neueste Erkenntnisse: Wie bringen wir Spitzenmedizin in die Region und welche Anwendungsbeispiele Künstlicher Intelligenz gibt es, die man schon jetzt einsetzen kann? Hinzu kommen State-of-the-art Sitzungen zu verschiedenen radiologischen Themen, wie $z$. B. der Abdomen- und der Mammadiagnostik.

\section{Sind auch wieder Veranstaltungen für den radiologischen Nachwuchs geplant?}

Der Kongress richtet sich mit seinem Programm grundsätzlich auch an unsere Kollegen und Kolleginnen in der Weiterbildung zum Radiologen oder einer Schwerpunktbezeichnung. Der RKR-Freitag bietet spezielle „Fit für den Facharzt“-Kurse, dieses Jahr liegt der Fokus auf der Neuroradiologie.

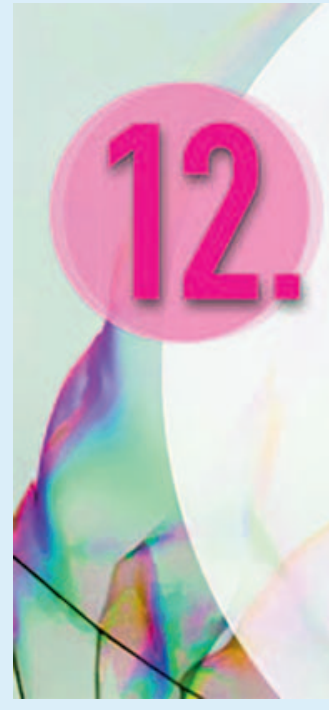

RADIOLOGIE $\square D$
KongressRUHR
7.-8. Nov. 2019

Kongresszentrum

Westfalenhallen Dortmund

www.radiologiekongressruhr.de
Haben Sie ein persönliches Highlight, das Sie auf gar keinen Fall verpassen möchten?

Ich freue mich auf viele spannende Vorträge, die ich auf keinen Fall verpassen möchte. Ich möchte nicht zu viel verraten, nur so viel: In jeder Sitzung haben sich die Vorsitzenden sehr viel Mühe mit der Themenaus- wahl gemacht - da sind einige echte Kracher dabei. Ganz besonders freue ich mich auf die zahlreichen interaktiven Sitzungen mit TED-Fragen - da kann man sein eigenes Wissen am besten überprüfen. 